\title{
Experimental and theoretical analysis of the intensity of beams diffracted by three-dimensional photonic crystals
}

\author{
Luis A. Dorado and Ricardo A. Depine* \\ Grupo de Electromagnetismo Aplicado, Departamento de Física, Facultad de Ciencias Exactas y Naturales, \\ Universidad de Buenos Aires, Buenos Aires, Argentina
}

Daniel Schinca

Centro de Investigaciones Ópticas (CIOp), (CIC-CONICET), La Plata, Argentina Dto Ciencias Básicas, Facultad de Ingeniería, Universidad Nacional de La Plata, Argentina

\author{
Gabriel Lozano and Hernán Míguez \\ Instituto de Ciencia de Materiales de Sevilla, Consejo Superior de Investigaciones Científicas, Sevilla, Spain
}

(Received 30 April 2008; revised manuscript received 18 June 2008; published 4 August 2008)

\begin{abstract}
An analysis of the diffracted beams emerging from three-dimensional photonic crystals is herein presented. The wave vectors of nonspecular beams are calculated for a triangular two-dimensional lattice and the change in their directions as a function of the wavelength is confirmed experimentally for the case of face-centeredcubic colloidal crystals illuminated under normal incidence. A fluctuating behavior of beam intensity as a function of the wavelength of the incident light is predicted for perfectly ordered lattices. As it is the case for specularly reflected and ballistically transmitted beams, this modulation arises from multipole resonances of the sphere ensemble that are smoothed out via the diffuse light scattering produced by imperfections in the crystalline structure. When optical extinction is introduced in order to model the effect of imperfections, it is possible to accurately reproduce experimental observations.
\end{abstract}

DOI: 10.1103/PhysRevB.78.075102

PACS number(s): 42.70.Qs, 41.20.Jb, 78.20.Bh, 78.40.-q

Three-dimensional (3D) photonic crystals, which are materials with a dielectric function having 3D spatial periodicity, have received much attention during the last decades mainly due to their potential applications in optical, infrared, and microwave devices. ${ }^{1,2}$ This kind of material is the only one capable of avoiding light propagation in all directions when the dielectric contrast is high enough, so they can present a complete band gap in their photonic band structure. ${ }^{3}$ This property has been used to mold the emission of optically active materials and proposed for several technological applications that are still under continuous research. ${ }^{4}$ The advent of fabrication techniques, that take advantage of the self-assembling properties of spherical colloidal particles in the micrometer scale, has made it possible to observe stop bands ${ }^{5}$ and even full gaps ${ }^{6}$ in the visible and near infrared spectra. Improvements in these techniques have led to high quality colloidal crystals with a low density of defects. $^{7-9}$ This has enabled the observation of previously undetected optical effects in the so-called high energy range, where the lattice constant is equal or greater than the wavelength. For this range, interesting fundamental phenomena have been observed when light propagates through low dispersion modes, such as the superprism effect ${ }^{10}$ or beam self-focusing. ${ }^{11}$ The optical spectra features observed in this range, such as the appearance of reflectance peaks and transmittance dips in the absence of any band gap, have generated an intense debate on the physical mechanisms originating these features. ${ }^{12-15}$

An interesting and almost unexplored phenomenon occurring in the high energy range is the opening of diffraction channels, ${ }^{13}$ that is, a finite number of diffracted beams emerge from the crystal slab when the photon energy is greater than a threshold energy or diffraction cutoff. These diffracted beams are propagating waves that can be projected on a screen in order to measure their intensities. However, up to date, most of the experimental and theoretical analyses in the high energy range available in the literature have been focused on the intensities of the specularly reflected and forwardly (or ballistically) transmitted beams under normal incidence. ${ }^{12}$ In this context, the physical origin of the features observed in the specular reflectance and forward transmittance spectra has recently been explained by means of multipole resonances inside the system. ${ }^{16}$ Besides, the modeling of disorder via extinction has also shown that sharp resonances are smoothed out more dramatically as a consequence of the diffuse light scattering. ${ }^{17,18}$ It has been found that the unintentional imperfections introduced in the fabrication process ${ }^{19}$ produce diffusely scattered light which removes energy from the coherent reflected and transmitted beams. ${ }^{16}$ This effect has been simulated by adding extinction to the theoretical model of a perfectly ordered lattice of spheres, obtaining fittings of the specular reflectance and forward transmittance experimental data with a degree of accuracy that has no precedent. ${ }^{17}$ Multipole resonances and extinction due to imperfections are expected to have similar effects for beams diffracted off normal. However, although their observation has been reported, ${ }^{13}$ no actual measurements of diffracted beam intensities, nor a theoretical prediction for them, has been performed so far.

In this paper, we present for the first time a complete description of nonspecular diffracted beams, both theoretically and experimentally. We measured the relative intensity of each diffracted spot as the wavelength (and therefore the diffraction angle) is varied in a laser beam emitted from an 
optical parametric oscillator that impinges perpendicularly on a self-assembled three-dimensional colloidal crystal. We use a Korringa-Kohn-Rostoker (KKR) approach to calculate the expected optical response of both perfect and imperfect (real) lattices. The effect of disorder is analyzed in detail and modeled by adding an imaginary part to the dielectric constant of the spheres. With extinction introduced in this fashion, we are able to reproduce the main features observed in our measurements. We find that perfectly ordered structures present a fluctuating spectral response whose optical features rapidly smoothens as extinction is introduced, although some features are robust against disorder and can be observed in both perfect and imperfect crystals.

Self-assembled 3D colloidal crystal films were prepared using the procedure described in Ref. 20 by the method of evaporation-induced self-assembly onto vertical or tilted substrates. In particular, they were deposited onto flat glass substrates by evaporation of polystyrene sphere suspensions (IKERLAT, polydispersity below $3 \%$, density $\rho=1.1 \mathrm{~g} / \mathrm{cm}^{3}$, refractive index $n=1.59$ ) of particle volume fraction comprised between $0.1 \%$ and $0.2 \%$. Evaporation temperature was kept constant for a given lattice growth process, and ranged between 35 and $50{ }^{\circ} \mathrm{C}$. As the suspension evaporates, a crystalline film is deposited on the substrate at the contact line with the suspension meniscus. The model structure is therefore a close-packed face-centered-cubic (fcc) lattice of spheres of dielectric constant $\varepsilon_{s}=2.53+i \varepsilon_{i}$ embedded in a medium of $\varepsilon=1$, which would correspond to latex spheres in air. The crystal-growth direction is typically the $[111],{ }^{21}$ so we will focus on the optical properties of diffracted beams when light impinges in that particular direction. Hence, the surface of the crystal slab presents a triangular lattice structure and subsequent layers are ordered in the known sequence $A B C A B C \ldots$, the incident light beam being normal to the slab surface (normal incidence). Particle diameter $d$ was measured from scanning electron microscopy images of such external surface. Average center to center distance between neighboring spheres was found to be $752 \mathrm{~nm}$. The light source was a tuneable optical parametric oscillator (OPO) pumped by the third harmonic $(355 \mathrm{~nm})$ of a Q-switched $\mathrm{Nd}$ :YAG $10 \mathrm{~Hz}$ repetition rate pulsed laser (Continuum Surelite II). As it is well known, nonlinear optical frequency mixing inside the beta-barium borate (BBO) crystal of the OPO produces coherent output at two frequencies (signal and idler) whose sum equals the pumping frequency for a certain propagation direction (phase matching condition). For 355 $\mathrm{nm}$ input wavelength, the signal fell in the visible range while the idler fell in the near infrared (NIR) range. Since the phase matching direction is frequency dependent, the output wavelength can be continuously varied by micromechanical tilt of the BBO. In our case, the IR idler output was blocked using an IR-absorbing visible-transmitting filter. Diffracted beam intensities were measured using a silicon photodiode attached to a $X Y Z \Theta \Phi$ mount that allowed us to accurately position the detector in the correct diffracted beam direction and angle. The photodiode output was fed into a digital oscilloscope. Although no time-resolved measurements were needed in this experiment, the $50 \Omega$ input coupling was selected to avoid any possible signal saturation. To minimize the effect of shot-to-shot fluctuation, intensity values were taken as the average of 32 shots. Since efficiency of nonspecular reflected beams was to be measured, a fraction of the incoming beam was taken using a calibrated beam splitter and its intensity measured with the same photodiode. Diffracted beam intensities for each selected wavelength were normalized to the corresponding incoming intensity, and reflectance (transmittance) was calculated in the usual way. The overall reflectance uncertainty was about $5 \%$.

The intensities of diffracted beams were calculated using the vector KKR method ${ }^{22,23}$ in its layer version. ${ }^{24}$ In this method, the crystal slab is first divided into layers parallel to a given crystallographic plane, each layer containing a twodimensional (2D) lattice of identical spheres. Next, a multipole expansion in spherical waves is used to calculate the multiple scattering between spheres in a given layer. Finally, a plane-wave expansion is used to account for the multiple scattering between layers. In all the calculations that follow, numerical convergence was obtained using a cutoff $L_{\mathrm{MAX}}$ $=9$ in the spherical wave expansion and 41 plane waves. ${ }^{25}$ If the sphere layers are parallel to the $x y$ plane, a set of $2 \mathrm{D}$ primitive lattice vectors is $\mathbf{a}_{1}=d \hat{\mathbf{x}}$ and $\mathbf{a}_{2}=d(\hat{\mathbf{x}}+\sqrt{3} \hat{\mathbf{y}}) / 2$, where $d$ is the distance between lattice sites, equal to the sphere diameter in a close-packed structure. The primitive reciprocal-lattice vectors can be chosen as $\mathbf{b}_{1}=4 \pi \hat{\mathbf{y}} /(\sqrt{3} d)$ and $\mathbf{b}_{2}=2 \pi(\hat{\mathbf{y}} / \sqrt{3}-\hat{\mathbf{x}}) / d$, then any reciprocal-lattice vector can be expressed as $\mathbf{g}=p \mathbf{b}_{1}+q \mathbf{b}_{2}$, where $(p, q)$ is a pair of integers. In normal incidence, when an incoming light beam travels in the positive $z$-axis direction, the wave vector of a diffracted beam emerging from the slab can be written as $\mathbf{K}_{\mathbf{g}}^{ \pm}=\mathbf{g} \pm \sqrt{k^{2}-|\mathbf{g}|^{2}} \hat{\mathbf{z}}$, where the $+(-)$ sign corresponds to a transmitted (reflected) beam, $k=2 \pi / \lambda$ is the magnitude of the incident wave vector and $\lambda$ is the wavelength. Each diffracted beam corresponds to a propagating wave if the $z$ component of $\mathbf{K}_{\mathrm{g}}^{ \pm}$is purely real. Then, diffraction channels are open when $|\mathbf{g}|<k$ and we have a diffraction cutoff whenever $|\mathbf{g}|=k$. For a triangular lattice, this condition reduces to

$$
\frac{d}{\lambda}=\frac{1}{n} \sqrt{p^{2}+\frac{(2 q+p)^{2}}{3}},
$$

where $n$ is the refractive index of the diffraction medium. In air $(n=1)$, six diffraction channels-corresponding to $(p, q)=(1,0),(1,-1),(0,-1),(-1,0),(-1,1),(0,1)$-open when $d / \lambda>2 / \sqrt{3} \cong 1.155$ or $a / \lambda>2 \sqrt{2} / \sqrt{3} \cong 1.633$, where $a$ is the lattice constant of the fcc conventional cubic cell. The wave vectors of these diffracted beams are located along a cone and the angle $\theta$ between $\mathbf{K}_{\mathbf{g}}^{ \pm}$and the $z$ axis is given by

$$
\sin (\theta)=\frac{2 \lambda}{\sqrt{3} d},
$$

which gives the angle $\theta$ of the first six diffracted beams as a function of the wavelength. The specularly reflected and forwardly transmitted beams correspond to channel $(0,0)$ which are always open channels. This situation is illustrated in Fig. 1 , where each reflected and transmitted beam is labeled according to the pair of integers $(p, q)$. The fact that the photonic crystal is supported on a glass substrate (refractive index $n=1.53$ ) introduces a first diffraction cutoff for transmitted beams at $a / \lambda=1.07$. Although those beams are 


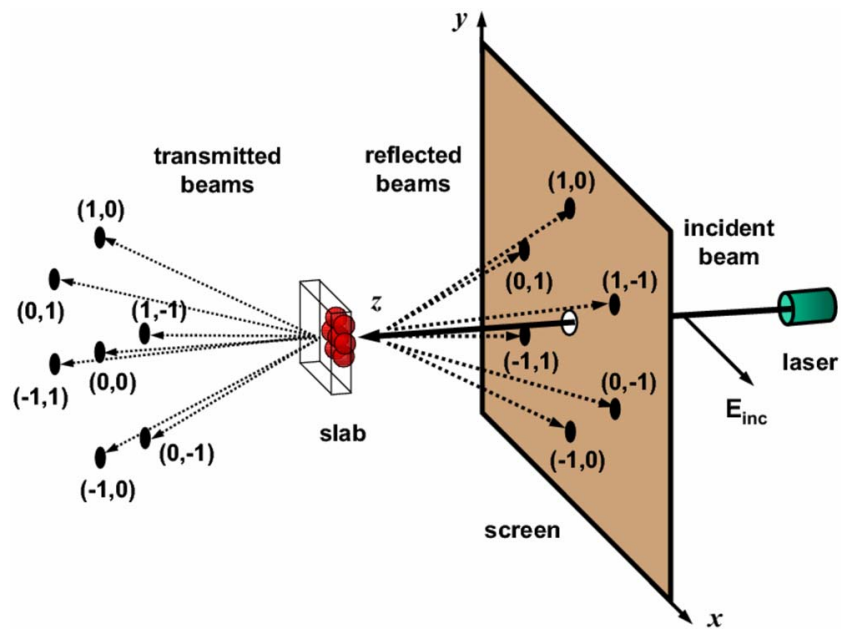

FIG. 1. (Color online) Scheme of the experimental setup used to measure the efficiency of nonspecular reflected beams. Transmitted beams are also indicated.

internally reflected at the glass-air surface and therefore do not propagate in air, they can be experimentally observed by frustrating the total reflection. ${ }^{13}$ The intensities of diffracted beams are referred to the incident-beam intensity. If we call $R_{(p, q)}$ and $T_{(p, q)}$ to the reflectance and transmittance coefficients of diffraction channel $(p, q)$, respectively, the total reflectance and transmittance will be $R=\Sigma_{(p, q)} R_{(p, q)}$ and $T$ $=\Sigma_{(p, q)} T_{(p, q)}$, where the sums include the open channel $(0,0)$. The coefficients $R_{(p, q)}$ and $T_{(p, q)}$ will also be referred to as efficiencies of channel $(p, q)$ in reflection and transmission, respectively. In the absence of energy losses, conservation of energy implies that $R+T=1$. Since disorder removes energy from the coherently scattered beams, it works as a sort of loss mechanism and we have $R+T<1$ in an actual experiment.

In Figs. 2(a) and 2(b), photographs of the diffraction spots can be observed on a screen for two different wavelengths,
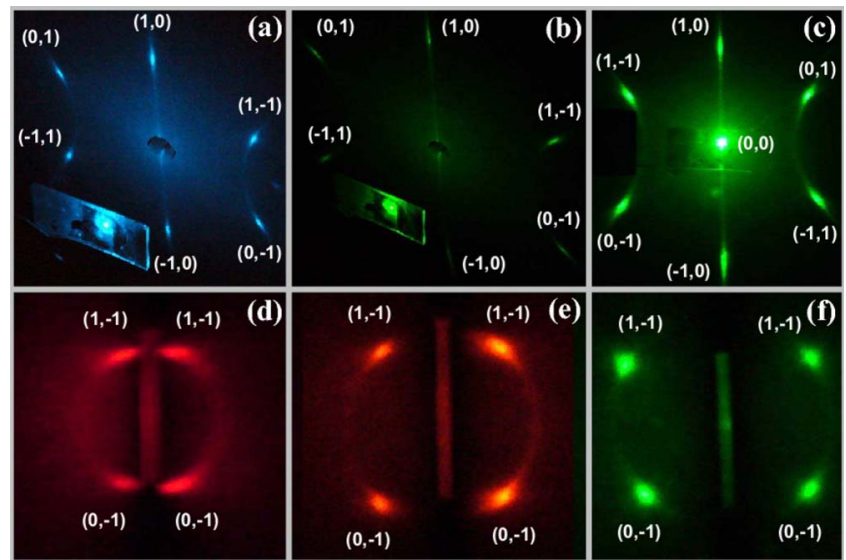

FIG. 2. (Color online) (a)-(b) Diffraction patterns of reflected beams projected on the screen shown in Fig. 1 ( $x y$ plane) for: (a) $\lambda=484 \mathrm{~nm}$ and (b) $\lambda=565 \mathrm{~nm}$. (c) Diffraction pattern of transmitted beams projected on a screen parallel to the $x y$ plane for $\lambda$ $=539 \mathrm{~nm}$. (d) - (f) Diffraction patterns of reflected and transmitted beams projected on a screen parallel to the $y z$ plane for: (d) $\lambda$ $=622 \mathrm{~nm}$, (e) $\lambda=593 \mathrm{~nm}$, and (f) $\lambda=512 \mathrm{~nm}$. namely, $\lambda=484 \mathrm{~nm}$ and $\lambda=565 \mathrm{~nm}$, respectively. These spots are produced by the six reflected beams that arise above the diffraction cutoff. The specularly reflected beam $(0,0)$ passes through a hole perforated in the screen to allow the laser beam reaching the crystal slab. Figure 2(c) shows the transmitted beams diffracted from the slab and projected on a screen also parallel to the $x y$ plane, as it is indicated in Fig. 1. In this case the wavelength is $\lambda=539 \mathrm{~nm}$ and the spot produced by the forwardly transmitted beam $(0,0)$ can be seen at the center of the picture. In these photographs, a uniform background due to diffuse light scattering can be observed. This energy is not captured by the detectors measuring the intensities of diffraction spots and then it can be considered as a kind of loss, which is simulated by adding extinction to the dielectric constant of the spheres in the lattice in our theoretical model. The existence of layers having a triangular lattice parallel to the $x y$ plane and piled up in the sequence $A B C A B C \ldots$ along the $z$ axis implies a symmetry relation for the efficiencies of diffracted beams when the incident light is polarized. In all the experiments, the incident wave is linearly polarized along the $x$ axis, as it is indicated by the incident electric-field vector $\mathbf{E}_{\text {inc }}$ in Fig. 1 . Under this condition, we have $R_{(1,0)}, R_{(1,-1)}=R_{(0,1)}, R_{(0,-1)}=R_{(-1,1)}$, and $R_{(-1,0)}$, thus there are essentially four different diffraction spots. A close look at Figs. 2(a)-2(c) allows us to observe this symmetry between spots.

Figures 2(d)-2(f) show photographs of diffraction spots obtained by using a screen parallel to the $y z$ plane for decreasing values of the wavelength, namely, 2 (d) $\lambda=622 \mathrm{~nm}$, 2(e) $\lambda=593 \mathrm{~nm}$, and 2(f) $\lambda=512 \mathrm{~nm}$. The width $(1 \mathrm{~mm})$ of the substrate onto which the sample (around $5 \mu \mathrm{m}$ wide) is deposited can be seen at the center of each picture and spots of reflected (transmitted) beams $(1,-1)$ and $(0,-1)$ are on the right (left) of the sample. As we have mentioned, diffraction spots of beams $(1,-1)$ and $(0,-1)$ have the same efficiencies as beams $(0,1)$ and $(-1,1)$, respectively. In Figs. 2(d)-2(f) we can see that the spots move away from the sample as the wavelength is decreased, following the change in the direction of the diffracted wave vectors $\mathbf{K}_{\mathrm{g}}^{ \pm}$predicted by Eq. (2). Substituting the experimental value attained for the sphere diameter $d=752 \mathrm{~nm}$ in Eq. (1) yields a diffraction cutoff wavelength $\lambda_{c}=651 \mathrm{~nm}$. Hence, Fig. 2(d) corresponds to a wavelength $(\lambda=622 \mathrm{~nm})$ close to the onset of diffraction spots and Eq. (2) gives $\theta$ near $90^{\circ}$, which is actually what we see in this figure. Thus, looking at the diffracted spots onto a screen perpendicular to the crystal provides a simple way of confirming experimentally the directional properties of the wave vectors of diffracted beams predicted by Eq. (2).

The calculated reflection efficiencies of diffracted channels are plotted in Fig. 3(a) as functions of the wavelength for a self-standing crystal 10 layers wide without extinction $\left(\varepsilon_{i}=0\right)$. The diffraction cutoff wavelength, $\lambda_{c}$, is indicated by the vertical dashed line. Note that diffraction efficiencies of nonspecular beams are all zero for wavelengths greater than $\lambda_{c}$. Although the curves present rapid fluctuations, two main peaks can be appreciated for all the efficiencies, one close to $\lambda=530 \mathrm{~nm}$ and another near $\lambda=475 \mathrm{~nm}$, particularly evident for the beam $(1,0)$. The angle $\theta$ of the diffracted beams, given by Eq. (2), is indicated in the upper horizontal scale, 


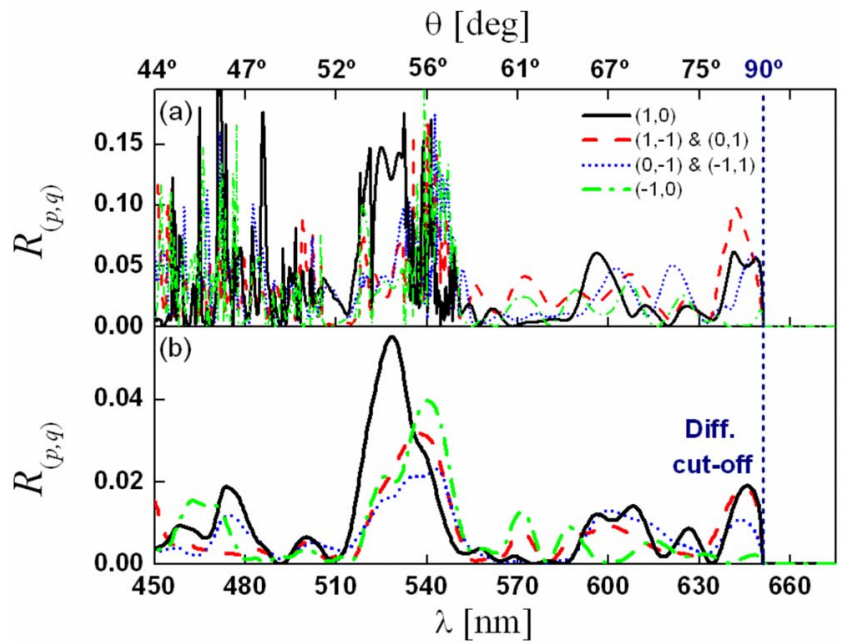

FIG. 3. (Color online) Calculated efficiencies of nonspecular reflected beams for (a) a perfect self-standing crystal 10 layers wide $\left(\varepsilon_{i}=0\right)$ and (b) the same lattice after introducing extinction $\left(\varepsilon_{i}\right.$ $=0.04)$.

where we can see that the main peak near $\lambda=530 \mathrm{~nm}$ corresponds to $\theta \cong 55^{\circ}$. Since each sphere in the lattice can be considered as a superposition of electric and magnetic dipoles, quadrupoles, octupoles, etc., these efficiency peaks originate from resonances inside the photonic crystal due to the interactions between multipoles. In other words, there is a natural resonance mechanism inside the crystal that is excited for only certain wavelengths. These results are consistent with previous observations and calculations that demonstrated that the origin of the optical response of the reflected and transmitted $(0,0)$ beams, observed for $a>\lambda$ in 3D photonic crystals, lies on multipolar resonances of the sphere ensemble. ${ }^{16}$ Hence it is not surprising that this mechanism also strongly affects the diffraction phenomena occurring in these lattices. In order to take into account the effect of disorder, the optical spectra of diffracted beams have been recalculated adding an imaginary part $\varepsilon_{i}=0.04$ to the dielectric constant of the spheres, which is a value that has provided good fitting with measurements of specular reflectance $\left(R_{(0,0)}\right)$ and forward transmittance $\left(T_{(0,0)}\right)$. The results are shown in Fig. 3(b), where a decrease of the intensities of the reflected beams is obtained as well as a smoothing of the curves compared with Fig. 3(a). Calculations have been performed for a glass-supported slab since real colloidal crystals are usually deposited on a glass substrate (refractive index $=1.53$ ). Nevertheless, we must point out that the reflected beam efficiencies of the glass-supported slab are basically the same as the ones obtained for the self-standing slab when extinction is introduced in the model. Furthermore, only the first six layers are involved in the optical response of the slab because waves have a finite penetration depth into the slab. ${ }^{16,18}$

Figure 4 shows the measured (solid lines) and calculated (dashed lines) efficiencies of the reflected beams $(1,0)$ and $(1,-1)$, where a good correlation between theory and experiment is obtained. In this case, a shift of the peaks toward higher wavelengths (red shift) can be appreciated. To fit the main peak positions of the experimental curves we have used

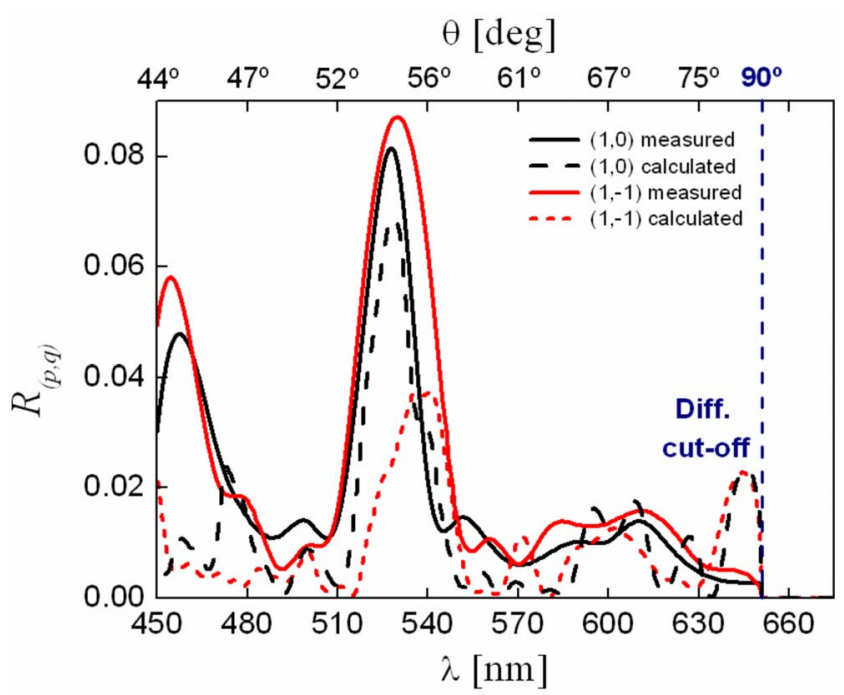

FIG. 4. (Color online) Measured (solid lines) and calculated (dashed lines, $\varepsilon_{s}=2.45+0.03 i$ ) reflection efficiencies of diffracted channels $(1,0)$ (black lines) and $(1,-1)$ (red lines).

$\varepsilon_{s}=2.45+0.03 i$. These results show that, although many fine spectral features are smoothed out due to disorder effects, some resonances are strong enough to survive the extinction process and can clearly be appreciated in a real experiment. At the same time, they confirm that extinction due to imperfections plays a crucial role in the optical response of colloidal crystal lattices and strongly determines the result of actual measurements.

In summary, we have predicted and observed a fluctuating optical response of nonspecular beams diffracted from perfectly ordered 3D photonic crystals, in good agreement with the behavior previously observed for reflected and transmitted beams. Diffracted beams in reflection and transmission have been recognized and labeled according to their associated reciprocal-lattice vectors, and the observed spectral dependence of the diffracted beams intensity has been accurately modeled using a KKR approach. The effect of disorder in the crystalline structure has been simulated by adding extinction to the theoretical model in order to reproduce the shape of the experimental curves. Finally, we have found some spectral features in diffracted beams arising from strong resonances that are robust against disorder effects.

\section{ACKNOWLEDGMENTS}

This work has been realized in the framework of a joint Spanish-Argentinian cooperation project CSIC-CONICET (Grant No. 2005AR0070). R.A.D. and L.D. acknowledge support from Consejo Nacional de Investigaciones Científicas y Técnicas (CONICET), Universidad de Buenos Aires (UBA) and Agencia Nacional de Promoción Científica y Tecnológica (BID 1728/OC-AR PICT-11-1785). D.S. aknowledges support from Grants No. PIP 5997 CONICET and No. PICT 26090 ANPCyT. H.M. is grateful for financial support from the Spanish Ministry of Science and Education under Grant No. MAT2005-03028. 
*rdep@df.uba.ar

${ }^{1}$ E. Yablonovitch, Phys. Rev. Lett. 58, 2059 (1987).

${ }^{2}$ K. Sakoda, Optical Properties of Photonic Crystals (SpringerVerlag, Berlin, 2001)

${ }^{3}$ K. M. Ho, C. T. Chan, and C. M. Soukoulis, Phys. Rev. Lett. 65 , 3152 (1990).

${ }^{4}$ J. D. Joannopoulos, R. D. Meade, and J. N. Winn, Photonic Crystals (Princeton University Press, Princeton, NJ, 1995).

${ }^{5}$ J. F. Bertone, P. Jiang, K. S. Hwang, D. M. Mittleman, and V. L. Colvin, Phys. Rev. Lett. 83, 300 (1999).

${ }^{6}$ A. Blanco, E. Chomski, S. Grabtchak, M. Ibisate, S. John, S. W. Leonard, C. Lopez, F. Meseguer, H. Míguez, J. P. Mondia, G. A. Ozin, O. Toader, and H. M. van Driel, Nature (London) 405, 437 (2000).

${ }^{7}$ H. Míguez, V. Kitaev, and G. Ozin, Appl. Phys. Lett. 84, 1239 (2004).

${ }^{8}$ S. Wong, V. Kitaev, and G. A. Ozin, J. Am. Chem. Soc. 125, 15589 (2003).

${ }^{9}$ K. Wostyn, Y. Zhao, B. Yee, K. Clays, A. Persoons, G. de Schaetzen, and L. Hellemans, J. Chem. Phys. 118, 10752 (2003).

${ }^{10}$ H. Kosaka, T. Kawashima, A. Tomita, M. Notomi, T. Tamamura, T. Sato, and S. Kawakami, Phys. Rev. B 58, R10096 (1998).

${ }^{11}$ A. Martínez, H. Míguez, A. Griol, and J. Martí, Phys. Rev. B 69, 165119 (2004).
${ }^{12}$ J. F. Galisteo-López and C. López, Phys. Rev. B 70, 035108 (2004).

${ }^{13}$ F. García-Santamaría, J. F. Galisteo-López, P. V. Braun, and C. López, Phys. Rev. B 71, 195112 (2005).

${ }^{14}$ X. Checoury, S. Enoch, C. López, and A. Blanco, Appl. Phys. Lett. 90, 161131 (2007)

${ }^{15}$ A. Balestreri, L. C. Andreani, and M. Agio, Phys. Rev. E 74, 036603 (2006)

${ }^{16}$ L. A. Dorado, R. A. Depine, G. Lozano, and H. Míguez, Opt. Express 15, 17754 (2007).

${ }^{17}$ L. A. Dorado, R. A. Depine, and H. Míguez, Phys. Rev. B 75 , 241101(R) (2007).

${ }^{18}$ L. A. Dorado, R. A. Depine, G. Lozano, and H. Míguez, Phys. Rev. B 76, 245103 (2007).

${ }^{19}$ G. Lozano and H. Míguez, Appl. Phys. Lett. 92, 091904 (2008).

${ }^{20}$ G. Lozano and H. Míguez, Langmuir 23, 9933 (2007).

${ }^{21}$ P. Jiang, J. F. Bertone, K. S. Hwang, and V. L. Colvin, Chem. Mater. 11, 2132 (1999).

${ }^{22}$ A. Modinos, Physica A 141, 575 (1987).

${ }^{23}$ K. Ohtaka, J. Phys. C 13, 667 (1980).

${ }^{24}$ N. Stefanou, V. Yannopapas, and A. Modinos, Comput. Phys. Commun. 113, 49 (1998); 132, 189 (2000).

${ }^{25}$ N. Stefanou, V. Karathanos, and A. Modinos, J. Phys.: Condens. Matter 4, 7389 (1992). 